\title{
In the Interactive Experience of The Elderly Tourism Product Design Style as the Center
}

\author{
Zhao $\mathrm{Yu}^{1, \text { a }}$ \\ ${ }^{1}$ Southeast University-Monash University Joint Graduate School , Southeast University, \\ Suzhou ,215123,China \\ aemail:yuzhao888999@163.com
}

Keywords: Interactive experience; The elderly; tourism products; design.

\begin{abstract}
With the method of "Interactive experience", according to the Elderly product market for the product opportunity gap, firstly determine the elderly travel product as the design direction by study; Secondly find elder people's travel problems by questionnaire survey method ; then design product gap as the elderly travel backpack, 'define the concept of specific product . In order to finding the solution considering the problems, eventually making the rough mode and product model.
\end{abstract}

\section{Introduction}

At present, Chinese has officially entered the "old age". China is not only the world's most populous country, but also the one with most aging population . In 2003 the total population of China has reached 1300000000, 60 years of age population and older reach (to) about $10 \%$ of the total about 130000000, The aging of the population in our country are put presnting unprecedented challenges to the whole society .

With the population aging, the development senile product still lags far behind in todays snile product market, the elderly supplies are monotone, the species are rare, give priority to with health care and medical products, products which are special for the elderly life, entertainment, transportations are less and less. Old people's special commodity according to their need are short, the elderly can not get enough respect and warm service. With the development of China's economy the improvement of living standards, when shopping and the establish and the perfecting of the old-age security system, products which the elderly demand have higher requirements therefore,we should paid attention to the elderly supplies design from each aspect of life of elderly people.

\section{Determine the product opportunity gap phase}

Interactive experience in new product development, is a method of products development, based on user's demands reguirment willing and aspirations of the development team stressed, and many other professional main stakeholders based. Mainly includes 4 stages: determine the product opportunity gap, understanding opportunity, product concept formation based on product opportunities and the realization of the concept. Firsty choose the old man for the target user group, and then find the old product opportunity gap [1]. Through the analysis on crowds at present related social - economic - technological factors, determining the new trend and create product opportunity gap.

According to the statistics of 2013 first half of the domestic travel information, relaxed leisure, affordable package is popular with elderly people.According to the show, as the result that they have more leisure time and a certain economic base, at the age of 45-64 years old elderly residents who are most interested in short-term tourism, the proportion of the total number of tourists in 45.7\%.we can summd up the following reasons of elderty people becoming the main factor , through the analysis of social - economic - technological factors (SET factors) , (L) the disposable income of the elderly more increaseing year by year; (2) having more leisure time; (3) the elderly 
needing emotional life; (4) the elderly and children living apart, their children can not accompany you around, the needing friends.

Then the opportunity gap can be set for the elderly tourism product design. The team conducted a questionnaire survey of 150 55----70 years old in the elderly, designed 20 questions around the tourism,according to the statistical results from the questionnaire, the elderly will encounter the following problems in travel: (1) the size are too big to take things; (2) the valuables safe problem (; 3) unexpected emergency situation;to have rest maybe happend (4) easy to forget the position of things ; (5) the sudden rain or snow. is not easy to control; (6) the back, waist and leg mere easily tired, and inconvenience .

\section{Understand the opportunities and the form of the concept stage}

The backpacker market survey

Through the analysis of the above problems, a design can help the elderly solve the problems of the backpack trip. At present, the backpack market mainly include two categories: professional types of backpacks,such as travel bag, knapsack and bag bicycle back rack; the common traveling bag types, bag and backpack bag. Through the comparative analysis on backpack market, backpack existing non is not suitable for the elderly, there are some defects.

At present, the backpack for the elderly, the main problems include: (1) professional travel Backpack have higr price, large volume, complex function,and are not suitable for the elderly; (2) the ordinary knapsack haven't partition or partition is not reasonable; (3) the ordinary backpack without the use of Population Division, is a pack of "fit" all the people, can not meet the special requirements of people; (4) the backpack design focus only on the appearance, they only have single function, and asy to take things.

Product definition

Through analyzing the elderly living conditions, travel problems they may encounter ,Backpack Travel analysis comparison, the design of the elderly travel backpack has a basic concept. This is a convenient backpack for old people to use, travel backpack concise, with certain functions: (L) having some simple and practical functions; (2) conforms to the physical characteristics of the elderly; (3) zoning clear easy to identify; (4) the soft material, strong.

The main problems of the elderly travel will hope to be solved through the backpack, such as not convenient rest, to have to take the backpack to take things often, backpack is not safe in the back, easy to forget the positions of things, easy fatigue backpack weight

The solution to the realization of product concept

Application of task analysis method, the problem is decomposed into a step by step manner, the results with the flow chart. Any product development goal is to reduce the need to complete the task of steps, or improve the efficiency of each step or simplify the difficulty, the effective degree is the original state and the present state of the effectiveness of [2] can be inspection of products.

For example, for the elderly often need to sit down and rest during the journey, to decompose the "sit" process, see figure 1 . Or sitting on the ground, or on +stone, if the weather is warm 


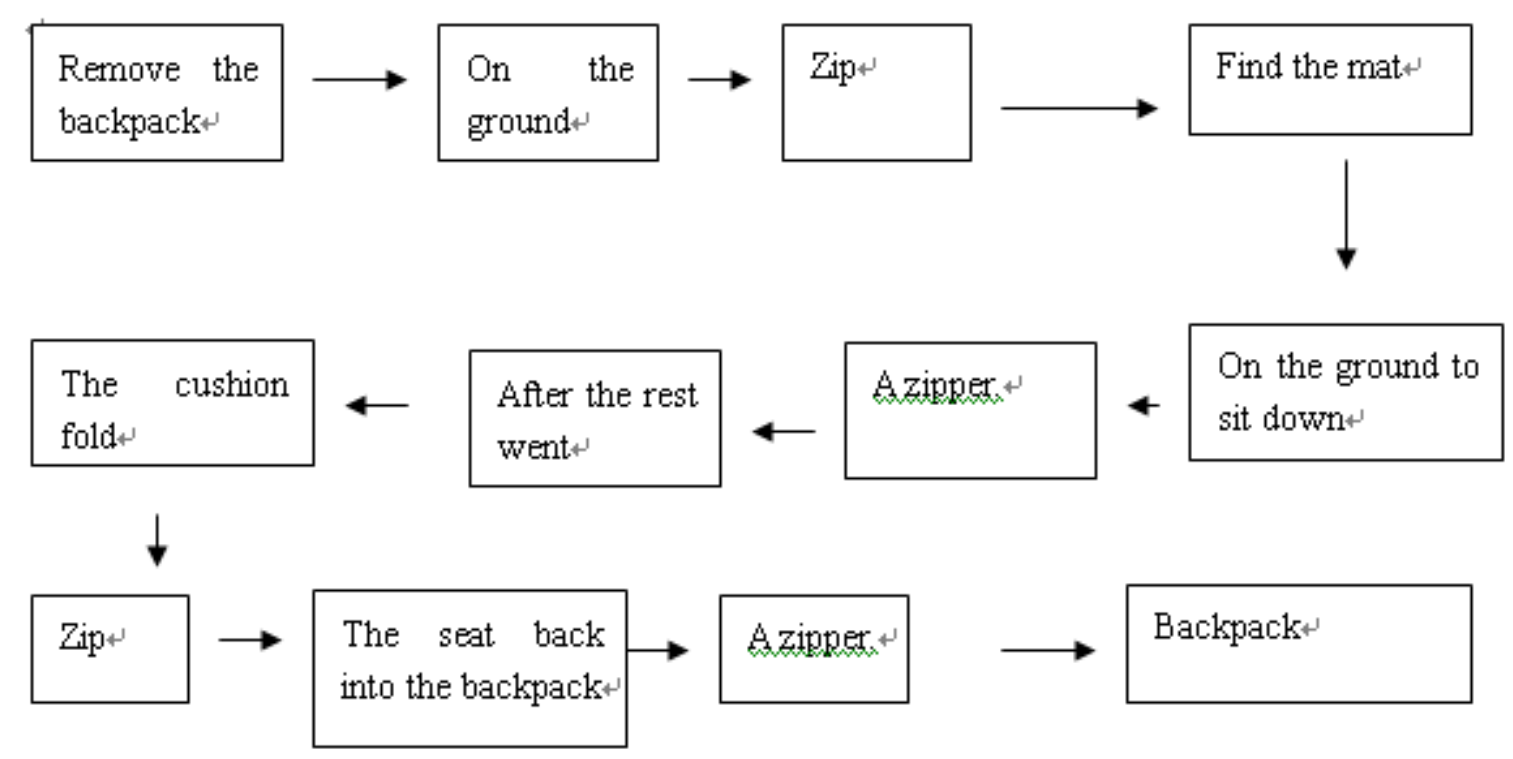

Fig.1 Decomposition chart of sitting

The warm, still can be accepted, but not HERSHEY'S weather conditions more trouble. Analysis found that the sit process is complicated, this should be a simplified process redesign, see figure 2 . Solution for the cushion with a backpack combine.

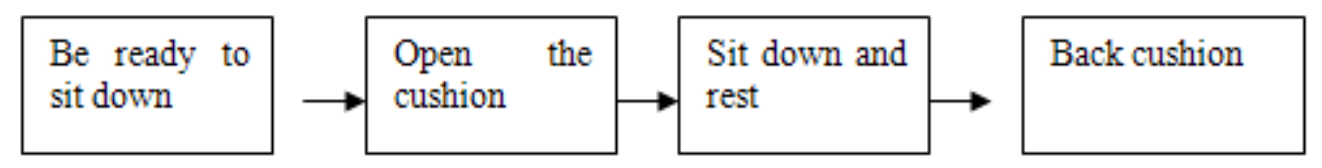

Fig.2 Reduced graph of sitting

In addition, decomposition process of unloading the backpack back her article

In addition, decomposition process of unloading the backpack back her articles, to find a solution to "backpack heavy is not easy to get items" problem. Such as the elderly medication process [3], see figure 3.

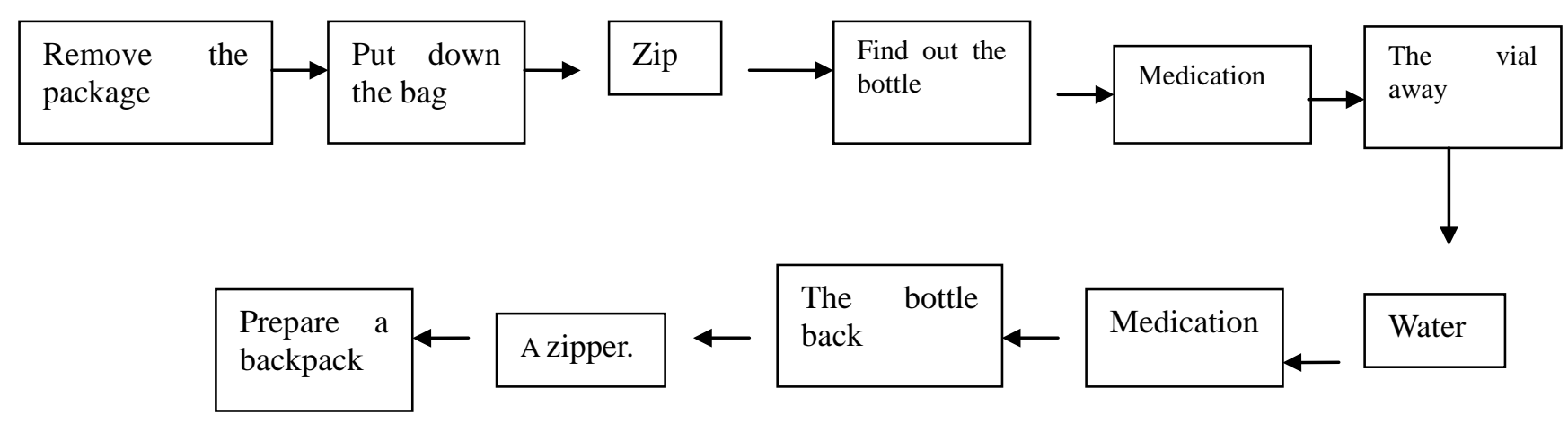

Fig.3 Derompositinn Chart of eating medicine

Through the analysis found that, even if it is a simple matter to the backpack off and back, for older travellers may be tricky. Also, easy to forget things, the elderly in the travel process, often forget small things in what position, often is the backpack every packet are searched to find what you want, it is very inconvenient for travellers. Solutions for the reasonable division sign to remind, backpack area, such as shoes and general supplies are placed separately.

Because will take some necessities for people when traveling, backpacking is heavy, and the travelling far way, heavy backpack on the shoulders and back pressure makes people easily fatigue, thus affecting the people travel mood. Method to solve through the rational use of materials, and the application of ergonomics, the package of gravity are dispersed or transfer, so as to reduce the 
pressure. Human factor analysis found the backpack, large small trapezoidal inclusion, can focus downward shift, thereby reducing pressure [4] shoulder. Human factor analysis found the backpack, large small trapezoidal inclusion, can focus downward shift, thereby reducing pressure [4] shoulder. The belt and the waist can protect the waist, but also transfer the shoulder pressure, and can solve the hips and waist support thin point combination of contradictions not firm.

Through the investigation and Analysis on the plan was finalized, has three main functions: (L) can alleviate fatigue, function; (2) simplified extract process, make the bag body can be split; (3) easy to find articles, reasonable division. These three features can be solved in a part of the problem in some of the elderly.

Aachieve the product concept phase

Next is the model making. The first production CAD drawings, determine the proportion and size, and then playing board, sewing bag, details of each model have batted [5]. After 2 times of grass, 2 formal model making, is finally showing old backpack design works as shown in figure 4 . This backpack

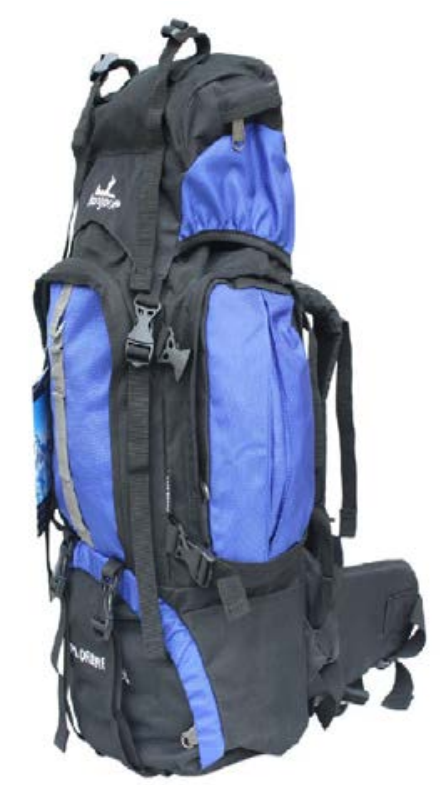

Fig.4 Backpac-k for the old

The design mainly includes the following 4 characteristics. (L) the bottom of the backpack space design. The bottom of the backpack space design into a triangular space used for shoes, and shoes triangular shape match, not only save space but also does not affect the upper space loading equipment items. (2) side pocket space design. Side pocket is used to store the drug and water bottles, side pocket opening is more convenient for the elderly to take. The elderly arm and arm flexibility drops, so should minimize the old arm too large swings. (3) the overall shape design. The backpack is under a big small obtrapezoid, can focus downward shift, thereby reducing the pressure, waist and waist design can protect the elderly waist. (4) design of multi functional bag. The backpack can be split into 2 package, will be valuable or commonly used goods forward transfer. There is a folding cushion the bottom of the backpack, which also housed a raincoat, and can be used as a cushion inner core and in the rainy day emergency. In a specific environment, multifunctional products are very popular with users, can solve the problem of emergency.

\section{Conclusions}

In the process of practice encountered many problems, the final results of the design there are many unsatisfactory, the beginning can be used to study the development of the elderly products, give some reference to other designers. Research and development for the elderly, reasonable function and excellent quality, for the development of enterprises has become the competition in the market opportunity for [6]. With the improvement of culture and education of the elderly, the elderly in consumer attitudes change more and more quickly, more and more old people spend more 
money for their own health and entertainment. This improves the elderly consumer purchasing power level to a certain extent, so designers should pay enough attention to the elder market.

\section{References}

[1] CAGAN J. Creating Breakthrough Products[M] .XIN Xiang - yang, Translate. Beijing : Machinery Industry Press, 2003.

[2] LIU Guan-zhong. Science of Human Affairs [M] .Changsha: South Central University Publishing House, 2006.

[3] ZHU Hui.Design and Research of Barrier Free Drug Packaging for the Old Men[J] .Packaging Engineering, 2006. 27(6): 293-295.

[4] LIN Ji-xiong .Anthropometric and Product Design [M]. Taibei: Taihao Press. 1984.

[5] JIAN Zhao-quan.lndustrial Design Methodology [M].Beijing: Beijing Institute of Technology Press, 2000.

[6] STAVRIANOS L S.A Global History [M] .DONG Shu - hui. 